\title{
Evidence for epistatic interactions in antiepileptic drug resistance
}

\author{
Myeong-Kyu Kim ${ }^{1}$, Jason H Moore ${ }^{2}$, Jong-Ki Kim ${ }^{1}$, Ki-Hyun Cho ${ }^{1}$, Yong-Won Cho ${ }^{3}$, Yo-Sik Kim ${ }^{4}$, \\ Min-Cheol Lee ${ }^{5}$, Young-Ok Kim ${ }^{6}$ and Min-Ho Shin $^{7}$
}

To investigate the epistatic interactions involved in antiepileptic drug (AED) resistance, 26 coding single-nucleotide polymorphisms (SNPs) were selected from 16 candidate genes. A total of 200 patients with drug-resistant localization-related epilepsy and 200 patients with drug-responsive localization-related epilepsy were genotyped individually for the SNPs. Rather than using the traditional parametric statistical method, a new statistical method, multifactor dimensionality reduction (MDR), was used to determine whether gene-gene interactions increase the risk of AED resistance. The MDR method indicated that a combination of four SNPs (rs12658835 and rs35166395 from GABRA1, rs2228622 from EAAT3 and rs2304725 from GAT3) was the best model for predicting susceptibility to AED resistance with a statistically significant testing accuracy of 0.625 $(P<0.001)$ and cross-validation consistency of $10 / 10$. This best model had an odds ratio of 3.68 with a significant $95 \%$ confidence interval of $2.32-5.85(P<0.0001)$. Our results may provide meaningful information on the mechanism underlying $A E D$ resistance and, to the best of our knowledge, this is the first report of evidence for gene-gene interactions underlying AED resistance.

Journal of Human Genetics (2011) 56, 71-76; doi:10.1038/jhg.2010.151; published online 2 December 2010

Keywords: drug resistance; epilepsy; epistasis; pharmacogenetics

\section{INTRODUCTION}

Although there have been numerous developments in antiepileptic drug (AED) therapy, epilepsy remains uncontrolled in a significant proportion of patients, even with appropriate polytherapy at maximal tolerated doses. ${ }^{1}$ However, in both refractory and responsive patients, the $\mathrm{AED}$ response of the individual patient is generally unpredictable. The biological basis of medical intractability, or pharmacoresistance, is poorly understood, but it is likely that, just as epilepsy per se is a heterogeneous condition with multiple etiologies, the pathogenesis of 'AED resistance' is multifactorial and may involve both genetic and environmental factors. ${ }^{2,3}$

There are numerous examples of cases in which interindividual differences in drug response are due to sequence variants in genes encoding drug-metabolizing enzymes, drug transporters or drug targets. ${ }^{4,5}$ Gene-gene interactions have been considered to be a primary mechanism underlying the pathogenesis of many common complex diseases or conditions. ${ }^{6-8}$ However, most candidate gene association studies on AED resistance have focused on an individual genetic variant with the potential main effect, and none of these studies could find evidence for a strong, single-gene effect on AED resistance. ${ }^{9-12}$ This is partially because of the limitations of parametric statistical methods for detecting gene effects that are solely or partially dependent on interactions with other genes. ${ }^{13,14}$ In this study, with the working hypothesis that AED resistance is a polygenic disorder in which several genetic variants with a modest effect interact with each other, we tried to find pharmacogenetic evidence of epistatic interactions underlying AED resistance using a new statistical method.

\section{SUBJECTS AND METHODS}

Subjects

Consecutive patients with an established clinical diagnosis of localizationrelated epilepsy, as defined by international guidelines, ${ }^{15}$ were recruited from epilepsy clinics at three regional tertiary hospitals in Korea. Table 1 summarizes the characteristics of the sample set. All study participants were eligible if they had drug-resistant (DR group) or drug-responsive (DS group) epilepsy according to the following definitions and criteria. Drug resistance was defined as the occurrence of at least four unprovoked seizures in the course of the year before recruitment, with trials of two or more appropriate AEDs at maximal tolerated doses, which were established on the basis of the occurrence of clinical side effects at supramaximal doses. Patients who underwent surgical treatment for drug-resistant epilepsy were classified as having drug-resistant epilepsy, regardless of the surgical outcome. However, patients who were frequently in poor compliance with AED therapy and those who had reported seizures with a questionable semiology were excluded from this study. In patients treated with single AED, drug responsiveness was defined as complete freedom from

${ }^{1}$ Department of Neurology, Chonnam National University Medical School, Gwangju, Korea; ${ }^{2}$ Computational Genetics Laboratory, Department of Genetics, Dartmouth Medical School, Lebanon, NH, USA; ${ }^{3}$ Department of Neurology, Dongsan Medical Center, Keimyung University, Daegu, Korea; ${ }^{4}$ Department of Neurology, Wonkwang University School of Medicine, Iksan, Korea; ${ }^{5}$ Department of Pathology, Chonnam National University Medical School, Gwangju, Korea; ${ }^{6}$ Department of Pediatrics, Chonnam National University Medical School, Gwangju, Korea and ${ }^{7}$ Department of Preventive Medicine, Chonnam National University Medical School, Gwangju, Korea

Correspondence: Dr M-K Kim, Department of Neurology, Chonnam National University Medical School, Hakdong 8, Donggu, Gwangju 501-757, Korea.

E-mail: mkkim@jnu.ac.kr

Received 18 July 2010; revised 11 October 2010; accepted 9 November 2010; published online 2 December 2010 
Table 1 Characteristics by case-control status

\begin{tabular}{lcc}
\hline & $\begin{array}{c}\text { Responsive group } \\
(\mathrm{n}=200)\end{array}$ & $\begin{array}{c}\text { Refractory group } \\
(\mathrm{n}=200)\end{array}$ \\
\hline $\begin{array}{l}\text { Gender } \\
\text { Man } n(\%)\end{array}$ & $101(50.5)$ & $97(48.5)$ \\
Age of seizure onset (years) & $17.31 \pm 13.2$ & $13.12 \pm 11.8$ \\
& & \\
Etiology of epilepsy (\%) & $86(43.0)$ & $158(79.0)$ \\
Symptomatic, focal & 54 & 108 \\
TLE & 32 & 50 \\
Non-TLE & $114(57.0)$ & $42(21.0)$ \\
Cryptogenic, focal & &
\end{tabular}

Abbreviation: TLE, temporal lobe epilepsy.

seizures for at least 1 year up to the date of the last follow-up visit. A total of 200 healthy volunteers without a family history of epilepsy were recruited in order to develop single-nucleotide polymorphisms (SNPs) of the candidate genes conferring AED resistance. The study was approved by the institutional review board at each of the three hospitals, and informed consent was obtained from all study participants.

\section{Candidate genes for AED response}

The candidate genes with a potential influence on AED response tested in this study were as follows: (1) genes encoding drug transporters, MDR1 ( $A B C B 1$ ), MRP2 ( $A B C C 2)$ and BCRP (ABCG2), as overexpression of these genes has been observed in the brains of patients with refractory epilepsy ${ }^{16,17}$ and (2) genes encoding drug targets such as voltage-gated neuronal sodium channels (SCN1A, SCN1B and SCN2A), potassium channels (KCNQ2, KCNQ3 and KCNQ5), calcium channels $(C A C N A 1 H)$, chloride channels (CLCN2), acetylcholine receptors (CHRNA4 and CHRNB2) and $\gamma$-aminobutyric acid (GABA) receptors (GABRA1 and GABRG2), as they are the main therapeutic targets of AEDs and are known to be causative of familial epilepsy syndromes, $10,18,19$ and (3) genes encoding neurotransmitter transporters (EAAT1, EAAT2, EAAT3, VGLUT1, VGLUT2, VGLUT3, GAT1 and GAT3), as they are the potential therapeutic targets of AEDs. ${ }^{20}$

\section{DNA pooling}

Genomic DNA was extracted from peripheral blood lymphocytes using a standard protocol. To screen common SNPs with a relatively high minor allele frequency in the candidate genes in a timely and cost-effective manner, a pool of DNA was established from 200 healthy DNAs, using the protocol recommended by the manufacturer (PicoGreen dsDNA Quantitation Reagent, Molecular Probes, Eugene, OR, USA). In brief, double-stranded DNAs were stained with an ultrasensitive fluorescent dye (PicoGreen dsDNA Quantitation Reagent, Molecular Probes), and the total amount of fluorescent-stained DNA in each individual was measured using a fluorometer (Picofluor Handheld Fluorometer, Molecular Probes). Quantification was performed in duplicate for each sample. An equal amount of DNA from 200 subjects per group was then mixed into a pool.

SNP development and estimation of their relative allele frequencies All coding regions of the 23 candidate genes, including the exon-intron boundary sequences of each candidate gene, were amplified using pooled DNA from the control group by PCR. Appropriate forward and reverse primer sets (data available on request) were prepared on the basis of GenBank sequences. PCR assays were carried out according to the method described elsewhere. ${ }^{21}$ Direct sequencing of both strands was performed using BigDye terminator kits (PE Biosystems, Foster City, CA, USA), and each electropherogram was analyzed visually using Chromas 2.13 (Technelysium, Queensland, Australia).

The relative allele frequencies for all SNPs determined in this study were estimated using the comparative method described elsewhere. ${ }^{21}$ To identify individual heterozygotes for the identified SNPs, 10 random DNA samples consisting of the pooled DNA were genotyped using the exact same PCR conditions as those used for the pooled DNA.

\section{Selection of SNPs and genotyping}

SNPs for genotyping were selected on the basis of the following criteria: (1) only exonic SNPs with an estimated minor allele frequency $>0.05$ of each gene were selected and (2) if there were two or more variants in a gene that were in complete linkage disequilibrium from each other, only one of them was randomly selected. Thus, a total of 26 SNPs that fulfill the selection criteria were selected from 16 candidate genes (Table 2).

A total of 200 patients with drug-resistant epilepsy (DR group) and 200 patients with drug-responsive epilepsy (DS group) were genotyped individually for 26 SNPs by PCR and direct sequencing analysis.

\section{Statistical analysis}

Case-control association study. Genotype frequencies at each locus were tested for the Hardy-Weinberg equilibrium. The strength of the association between single SNPs and AED pharmacoresistance was evaluated as an odds ratio, according to the mode of inheritance of a causal allele obtained with HAPSTAT 3.0 (University of North Carolina, Chapel Hill, NC, USA). The differences were considered significant for $P$-values of $\leqslant 0.05$. The mode of inheritance can be additive, dominant or recessive. Under the additive model, two copies of a causal allele have twice the effect on the trait as compared with a single copy. Under the dominant model, having one or two copies has the same effect. Under the recessive model, having only two copies of the causal allele will affect the trait.

Multifactor dimensionality reduction analysis. Gene-gene interactions were analyzed using the four-step process outlined by Moore et al. ${ }^{22}$ First, noisy SNPs were removed from the pool of possible candidates by using the Tuned ReliefF (TuRF) filter algorithm developed by Moore and White. ${ }^{23}$ The TuRF algorithm systematically removes attributes that have low weight in situations in which the number of noisy attributes is large so that the weights of the remaining attributes can be reestimated. Using this procedure, we selected the top four SNPs as we only wanted to evaluate the best two-way, three-way or four-way gene-gene interactions. This decision was made to reduce the chance of overfitting the data, which is possible when considering high-order interactions (for example, $>4$ ) and many different SNP combinations in relatively small data sets. We then confirmed that the TuRF scores of all four SNPs selected for each analysis were positive or close to zero, indicating that they each have genotypes that are more similar within the case and control groups than between the groups. SNPs with negatives scores were eliminated during this filter step and thus were not considered in the multifactor dimensionality reduction (MDR) analysis. Second, we constructed all possible combinations of two, three and four polymorphisms using the MDR constructive induction algorithm. ${ }^{7}$ Third, using the MDR kernel, we created a new multilocus variable with two levels (high and low risk) by pooling multilocus genotypes. The procedure recodes the data to allow easier detection of interactions. We then used a naive Bayes classifier in the context of 10 -fold cross-validation to estimate the balanced testing accuracy ${ }^{24}$ of each one-dimensional MDR-coded variable of the best two-, three- and four-factor model. The single best model that maximized the testing accuracy was selected, as this is the model that is most likely to generalize to independent data sets. We also report the crossvalidation consistency that measures the number of times out of 10 divisions of data that the same best model was found. Statistical significance was evaluated using a 1000-fold permutation test to compare the observed testing accuracies with those expected under the null hypothesis of no association. Permutation testing corrects for multiple testing by repeating the entire analysis on 1000 data sets that are consistent with the null hypothesis. Models were considered significant at a type I error rate of 0.001 . Finally, as described by Moore et al., ${ }^{22}$ we used measures of interaction information to provide a statistical interpretation of the gene-gene interaction models. Interaction graphs were used to visualize the nature of the dependencies using version 1.2 of the open-source MDR software, which is freely available from www.epistasis.org. 
Table 2 Characteristics of the candidate single-nucleotide polymorphisms (SNPs)

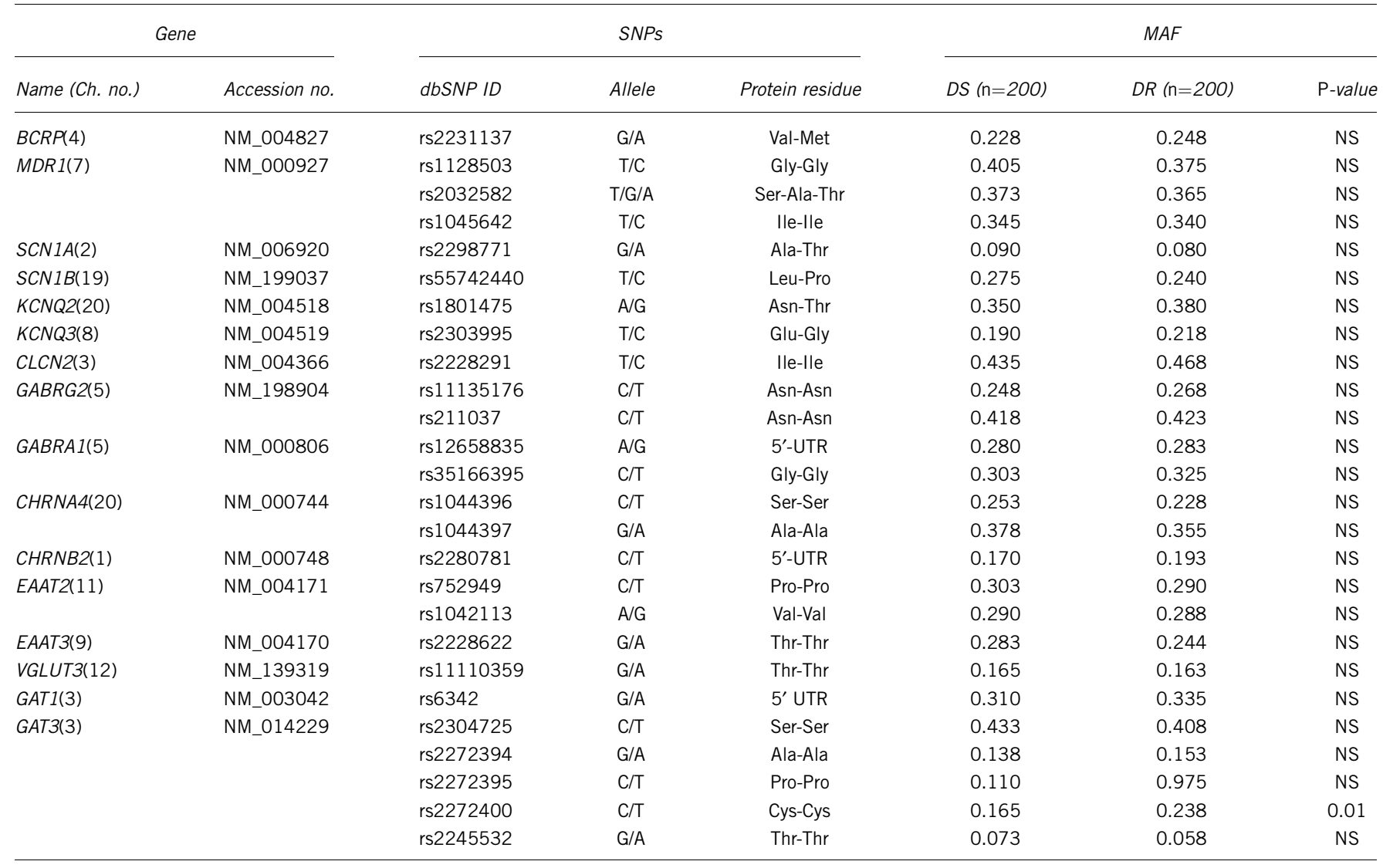

Abbreviations: Ch. no., chromosome number; DR, drug-refractory group; DS, drug-responsive group; MAF, minor allele frequency; NS, not significant.

\section{RESULTS}

Single-locus analysis

All 26 sites were in Hardy-Weinberg equilibrium in 400 patients with epilepsy. However, rs1128503 was out of Hardy-Weinberg equilibrium in the DR group, as was rs2228291 in the DS group, which may be indicative of weak single-locus effects. Nevertheless, there were no significant differences in genotype for any of the two loci between cases and controls (Table 2). The risk of susceptibility to AED resistance in patients with the mutant allele of each SNP, except rs2272400, was not significant when compared with that of the wildtype allele (Table 2). SNP rs2272400 (GAT3 c.1572 C>T) was nominally associated with AED pharmacoresistance $\left(P_{\text {allelic }}=0.01\right)$. The odds ratio for AED pharmacoresistance was 1.6 (95\% confidence interval (CI), 1.11-2.24; $P=0.01)$ and 1.5 (95\% CI, 1.03-2.23; $P=0.036)$ in the additive and dominant models of inheritance, respectively, when considering the minor allele $(\mathrm{T})$ as a causal allele (in preparing copy). Therefore, SNP rs2272400 was excluded from the MDR analysis with which we intended to find an evidence for epistatic interaction among genetic variants with modest effects that could not be detected by classical parametric statistical methods.

\section{MDR analysis}

The three-locus genotypes were recoded as binary data to reduce the dimensionality of high-order interaction among 25 SNPs tested: the heterozygote and minor allele homozygote as 1 and the major allele homozygote as 0 .

The four best SNPs selected by the TuRF filter algorithm were as follows: rs12658835 and rs35166395 (GABRA1), rs2228622 (EAAT3)
Table 3 Summary of MDR analysis results

\begin{tabular}{lrrc}
\hline Model & TA & CVC & P-value \\
\hline rs2228622 & 0.4800 & $6 / 10$ & NS \\
rs12658835, rs35166395 & 0.6075 & $10 / 10$ & $<0.001$ \\
rs12658835, rs35166395, rs2304725 & 0.5950 & $7 / 10$ & $\mathrm{NS}$ \\
rs12658835, rs35166395, rs2304725, rs2228622 & 0.6250 & $10 / 10$ & $<0.001$
\end{tabular}

Abbreviations: CVC, cross-validation consistency; MDS, multifactor dimensionality reduction; NS, not significant; TA, testing accuracy.

and rs2304725 (GAT3). Table 3 summarizes the results of the exhaustive MDR analysis, which evaluated all possible combinations of these four polymorphisms. The best model of each order is shown along with its testing accuracy, cross-validation consistency and significance level, as determined by permutation testing. The overall best model with the highest testing accuracy consisted of rs12658835, rs35166395, rs2228622 and rs2304725. This model had a statistically significant testing accuracy of $0.625(P<0.001)$, and a cross-validation consistency of $10 / 10$. This best model had an odds ratio of 3.68 , with a significant 95\% CI of 2.32-5.85 $(P<0.0001)$. Figure 1a summarizes the distribution of cases and controls for each of the 16 two-locus genotype combinations. Note that the pattern of high-risk (dark gray) and low-risk (light gray) genotype combinations is nonlinear across each multilocus dimension, which is evidence of a gene-gene interaction or epistasis.

Figure $1 \mathrm{~b}$ illustrates an interaction dendrogram that summarizes the estimates of interaction information (that is, entropy) for each pair 


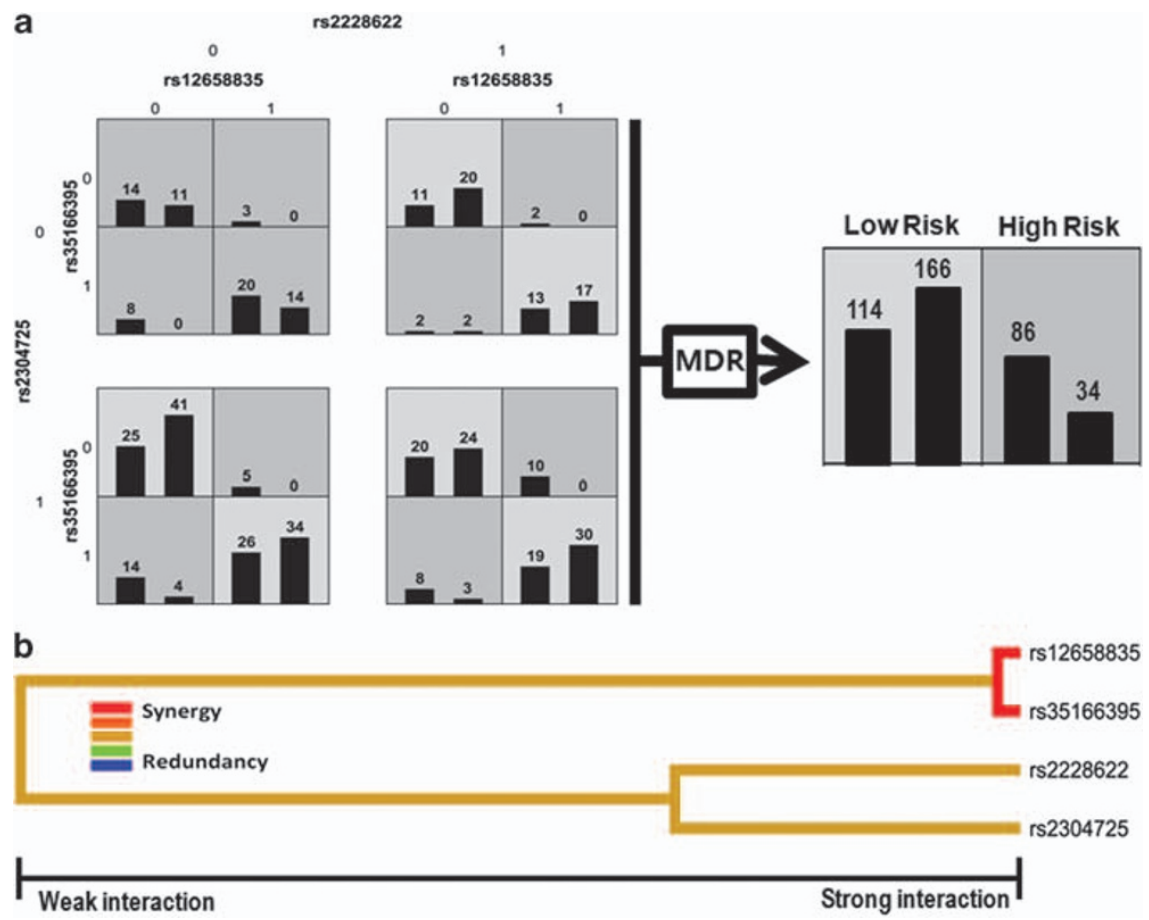

Figure 1 (a) Distribution of cases and controls across 16 two-locus genotype combinations. Each multilocus genotype combination is considered high risk when the ratio of cases to controls exceeds a threshold, $T$, which is equal to the ratio of cases to controls in each population. The cell is classified as low risk if the ratio of cases to controls does not exceed the threshold T. High-risk combinations are depicted as dark-shaded cells; low-risk combinations are depicted as light-shaded cells; and empty cells are left blank. For each cell, the left bar represents cases, and the right bar represents controls. The pattern of high-risk and low-risk cells differs across each multilocus dimension, and this is evidence of gene-gene interaction or epistasis. Also shown is the single multifactor dimensionality reduction (MDR) variable. (b) Interaction dendrogram for the four polymorphisms modeled by MDR. Note the synergistic (that is, non-additive) effects of single-nucleotide polymorphisms rs12658835 and rs35166395.

of SNPs considered. Shorter lines connecting two SNPs were indicative of a stronger interaction or dependency. A red or orange line connecting two polymorphisms suggests a positive information gain, which can be interpreted as a synergistic or non-additive relationship, whereas a blue or green line suggests a loss of information, which can be interpreted as redundancy or correlation (for example, linkage disequilibrium). A yellow line indicates independence or additivity. The interaction information analysis indicates that rs12658835 and rs35166395 interact in a synergistic manner, whereas the effect of rs2228622 or rs 2304725 is mostly independent. Thus, our four-locus MDR model can be decomposed into one synergistic effect and two independent effects.

\section{DISCUSSION}

The identification and characterization of a genetic predictor of AED resistance has been a long-felt need for both epileptologists and patients with refractory epilepsy. Indeed, AED resistance is especially suitable for pharmacogenetic investigation in view of the high prevalence of this disorder, the wide variety of individual responses to AEDs and the readily quantified outcomes of seizure control. ${ }^{25}$ Nevertheless, numerous attempts to find the genetic predictor of AED resistance conducted within the last decade turned out to be unsuccessful. ${ }^{10,12}$ This frustrating outcome should be considered, in a sense, as the result of a failure of strategy in which the working hypothesis seeks a single genetic variant with a main effect for a potentially polygenic disorder, as well as statistical limitations. It is becoming increasingly evident that many common human diseases cannot be attributed to a single gene or exposure factor. ${ }^{26}$
Several phenomena, such as phenocopy, trait heterogeneity, locus heterogeneity, plastic reaction norms (gene-environment interaction) and epistasis (gene-gene interaction), may greatly complicate the genetic architecture of biomedical traits, such as disease susceptibility. ${ }^{27}$ Epistasis is believed to have an important role in the genetic architecture of many common human diseases, ${ }^{13,27-29}$ but it is difficult to detect statistical patterns of epistasis in human populations because of the sparseness of available data in multiple dimensions. The combination of data sparseness and nonlinearity in the relationship between genotype and phenotype has motivated the development of multiple analytical approaches, including MDR. The MDR method was developed as a non-parametric and genetic model-free datamining strategy for identifying combinations of SNPs and other discrete factors, such as smoking, that are predictive of a discrete clinical end point. This method has been successfully used to detect gene-gene and gene-environment interactions for a variety of clinical end points, including Alzheimer's disease, asthma, atrial fibrillation, autism, bladder cancer, hypertension, multiple sclerosis, myocardial infarction, schizophrenia and type II diabetes. ${ }^{24}$ The MDR method was also successfully used to detect evidence of epistasis in AED response in this study. Although the independent contribution of each genetic variant, rs12658835, rs35166395, rs2228622 or rs2304725, to AED resistance, if any, is too weak to be detected, epistatic interactions among these SNPs seemed to contribute significantly to susceptibility to AED resistance.

Thus far, two main hypotheses have been proposed to account for pharmacoresistant epilepsy: the transporter and target hypotheses. The transporter hypothesis contends that pharmacoresistance arises 
because AEDs do not gain access to their sites of action in the brain. This phenomenon is thought to be caused by overexpression of drug efflux transporter genes, such as MDR1 at the blood-brain barrier, that limit AED access to the brain. ${ }^{16,17}$ The target hypothesis, on the other hand, contends that target receptor sites are somehow altered in the epileptic brain so that they are much less sensitive to the anticonvulsant effects of systemically administered drugs. So far, a reduction in drug target sensitivity in chronic human and experimental epilepsy has been suggested for the voltage-gated sodium channel and the GABA-A receptor. ${ }^{30}$ Previously, we failed to demonstrate a definite evidence of epistasis in AED resistance among the genes encoding voltage-gated sodium channels, SCN1A, SCN1B and SCN2A. ${ }^{31}$ Instead, in this study, the genes encoding GABA receptor 1 subunit (GABRA1) and both excitatory and inhibitory neurotransmitter transporters (EAAT3 and GAT3, respectively) turned out to be minor contributors to epistasis in AED resistance. Especially, a strong synergistic interaction was found between two SNPs in the same gene, SNP rs12658835 in the $5^{\prime}$ untranslated region and SNP rs35166395 in exon 4 of the GABRA1 gene. Given that the two SNPs were not in linkage disequilibrium as depicted in Figure $1 \mathrm{~b}$ and the MDR result from interaction between the two SNPs passes the permutation test at an $\alpha$ of 0.001 (Table 3), it would be possible that certain genotypic combinations of the two SNPs mainly contribute to susceptibility to AED resistance in this four-SNP interaction. In addition to this synergistic effect, each effect of rs2228622 (EAAT3) or rs2304725 (GAT3) may also contribute to susceptibility to AED resistance in an independent manner.

GABA-A receptor 1 subunit is an essential component of the GABA-A receptor, in which GABA binds to exert the main fast inhibitory neurotransmission in the mammalian brain. Genetic studies in humans reveal a range of idiopathic generalized epilepsy syndromes linked to mutations in the GABA-A receptor 1 subunit gene (GABRA1). GABAergic neurotransmission is terminated by the uptake of GABA into the presynaptic terminal and the surrounding astroglial cells by sodium-dependent transporters, such as GAT3 (human SLC6A11). EAAT3 (human SLC1A1) is a high-affinity glutamate transporter that has an essential role in transporting glutamate across plasma membranes. In brain, the transporter is crucial in terminating the action of the excitatory neurotransmitter glutamate. ${ }^{20}$ Therefore, on the basis of the results from this study, it can be inferred that the excitatory/inhibitory imbalance in the epileptic brain, resulting from alterations in both ambient glutamate/GABA concentration and their receptor sites, may underlie the pathogenesis of AED resistance.

There are several limitations to this study. First, we adopted a timely and cost-effective screening method for developing putative candidates as described above, in which the SNPs with a lower minor allele frequency could not be detected. Second, the promoter region of the candidate genes was excluded from SNP development because its exact boundary in DNA was not clear for many candidate genes. Third, although they are coding SNPs, the intrinsic value of the majority of the candidate SNPs is not known yet. Fourth, the number and characteristics of the SNPs tested may be not sufficient to support the intrinsic value of the MDR method in dissecting the gene-gene interactions that underlie AED resistance. This means that, in certain circumstances, an SNP or SNPs with a stronger effect than the SNPs tested in this study can be obtained in the corresponding gene or new candidate genes in future studies. Fifth, association studies have the intrinsic problem of returning false-positive results when considering high-order interactions and many different SNP combinations in relatively small data sets, as was the case in this study. To address this problem, we adopted a newer statistical technique to control for bias and multiple testing, which has been successfully used before to detect epistatic interactions for common complex disorders. ${ }^{24}$ However, despite these limitations, evidence of epistasis in AED resistance was found in this study and, to the best of our knowledge, this is the first report of gene-gene interactions underlying AED resistance.

Our results might not be conclusive; nevertheless, we believe that they might make a meaningful suggestion about the pathomechanism that underlies AED resistance.

\section{CONFLICT OF INTEREST}

The authors declare no conflict of interest.

\section{ACKNOWLEDGEMENTS}

This study was supported by a grant from the Korea Health 21 R\&D Project, Ministry of Health, Welfare \& Family Affairs, Republic of Korea (A080307) and by a grant (CRI-08016-1) from the Chonnam National University Hospital Research Institute of Clinical Medicine.

\section{DISCLAIMER}

We confirm that we have read the Journal's position on issues involved in ethical publication and affirm that this report is consistent with those guidelines.

1 Kwan, P. \& Brodie, M. J. Early identification of refractory epilepsy. N. Engl. J. Med. 342, 314-319 (2000)

2 Regesta, G. \& Tanganelli, P. Clinical aspects and biological bases of drug-resistant epilepsies. Epilepsy Res. 34, 109-122 (1999).

3 Kwan, P. \& Brodie, M. J. Refractory epilepsy: a progressive, intractable but preventable condition? Seizure 11, 77-84 (2002).

4 Evans, W. E. \& Relling, M. V. Pharmacogenomics: translating functional genomics into rational therapeutics. Science 286, 487-491 (1999).

5 McLeod, H. L. \& Evans, W. E. Pharmacogenomics: unlocking the human genome for better drug therapy. Annu. Rev. Pharmacol. Toxicol. 41, 101-121 (2001).

6 Gibson, G. Epistasis and pleiotropy as natural properties of transcriptional regulation. Theor. Popul. Biol. 49, 58-89 (1996).

7 Ritchie, M. D., Hahn, L. W., Roodi, N., Bailey, L. R., Dupont, W. D., Parl, F. F. et al. Multifactor-dimensionality reduction reveals high-order interactions among estrogenmetabolism genes in sporadic breast cancer. Am. J. Hum. Genet. 69, 138-147 (2001).

8 Williams, S. M., Ritchie, M. D., Phillips, J. A. III., Dawson, E., Prince, M., Dzhura, E. et al. Multilocus analysis of hypertension: a hierarchical approach. Hum. Hered. 57, 28-38 (2004).

9 Siddiqui, A., Kerb, R., Weale, M. E., Brinkmann, U., Smith, A., Goldstein, D. B. et al. Association of multidrug resistance in epilepsy with a polymorphism in the drugtransporter gene ABCB1. N. Engl. J. Med. 348, 1442-1448 (2003).

10 Depondt, $C$. The potential of pharmacogenetics in the treatment of epilepsy. Eur. J. Paediatr. Neurol. 10, 57-65 (2006).

11 Kim, Y. O., Kim, M. K., Woo, Y. J., Lee, M. C., Kim, J. H., Park, K. W. et al. Single nucleotide polymorphisms in the multidrug resistance 1 gene in Korean epileptics. Seizure 15, 67-72 (2006).

12 Shahwan, A., Murphy, K., Doherty, C., Cavalleri, G. L., Muckian, C., Dicker, P. et al. The controversial association of ABCB1 polymorphisms in refractory epilepsy: an analysis of multiple SNPs in an Irish population. Epilepsy Res. 73, 192-198 (2007).

13 Templeton, A. R. in Epistasis and The Evolutionary Process (eds. Wade, M., Brodie, B. III \& Wolf, J.) 41-57 (Oxford Univ. Press, Oxford, 2000).

14 Glazier, A. M., Nadeau, J. H. \& Aitman, T. J. Finding genes that underlie complex traits. Science 298, 2345-2349 (2002)

15 Commission on Classification and Terminology of the International League Against Epilepsy. Proposal for revised classification of epilepsies and epileptic syndromes. Epilepsia 30, 389-399 (1989).

16 Loscher, W. \& Potschka, H. Role of multidrug transporters in pharmacoresistance to antiepileptic drugs. J. Pharmacol. Exp. Ther. 301, 7-14 (2002).

17 Lazarowski, A., Czornyj, L., Lubienieki, F., Girardi, E., Vazquez, S. \& D’Giano, C. ABC transporters during epilepsy and mechanisms underlying multidrug resistance in refractory epilepsy. Epilepsia 48 (Suppl 5), 140-149 (2007).

18 McNamara, J. O. in Goodmann \& Gilman's The Pharmacological Basis of Therapeutics (eds. Hardman, J.G., Limbird, L.E., \& Gilman, A.G.) 521-547 (McGraw-Hill, New York, 2001).

19 Gardiner, M. Genetics of idiopathic generalized epilepsies. Epilepsia 46 (Suppl 9), 15-20 (2005)

20 Meldrum, B. S. \& Rogawski, M. A. Molecular targets for antiepileptic drug development. Neurotherapeutics 4, 18-61 (2007). 
21 Kim, M. K., Nam, T. S., Choi, K. H., Jang, S. Y., Kim, Y. O. \& Lee, M. C. Usefulness of direct sequencing of pooled DNA for SNP identification and allele-frequency determination compatible with a common disease/common variant hypothesis. Genet. Mol. Res. 9, 772-779 (2010).

22 Moore, J. H., Gilbert, J. C., Tsai, C. T., Chiang, F. T., Holden, W., Barney, N. et al. A flexible computational framework for detecting, characterizing, and interpreting statistical patterns of epistasis in genetic studies of human disease susceptibility. J. Theor. Biol. 241, 252-261 (2006).

23 Moore, J. H., White, B. C. in Lecture Notes in Computer Science (eds. Rajapakse, J. C. et al.) 166-175 (Springer, New York, 2007).

24 Velez, D. R., White, B. C., Motsinger, A. A., Bush, W. S., Ritchie, M. D., Williams, S. M. et al. A balanced accuracy function for epistasis modeling in imbalanced datasets using multifactor dimensionality reduction. Genet. Epidemiol. 31, 306-315 (2007).
25 Szoeke, C. E., Newton, M., Wood, J. M., Goldstein, D., Berkovic, S. F., Obrien, T. J. et al. Update on pharmacogenetics in epilepsy: a brief review. Lancet Neurol. 5, 189-196 (2006).

26 Pharoah, P.D., Dunning, A.M., Ponder, B.A. \& Easton, D.F. Association studies for finding cancer-susceptibility genetic variants. Nat. Rev. Cancer 4, 850-860 (2004).

27 Thornton-Wells, T. A., Moore, J. H. \& Haines, J. L. Genetics, statistics and human disease: analytical retooling for complexity. Trends Genet. 20, 640-647 (2004).

28 Cordell, H. J. Epistasis: what it means, what it doesn't mean, and statistical methods to detect it in humans. Hum. Mol. Genet. 11, 2463-2468 (2002).

29 Moore, J. H. The ubiquitous nature of epistasis in determining susceptibility to common human diseases. Hum. Hered. 56, 73-82 (2003)

30 Beck, H. Plasticity of antiepileptic drug targets. Epilepsia 48 (Suppl 1), 14-18 (2007).

31 Jang, S. Y., Kim, M. K., Lee, K. R., Park, M. S., Kim, B. C., Cho, K. H. et al. Gene-togene interaction between sodium channel-related genes in determining the risk of antiepileptic drug resistance. J. Korean. Med. Sci. 24, 62-68 (2009). 